\title{
Representaciones sociales de la maternidad de mujeres jóvenes de Lima
}

\section{Kelly Cieza Guevara}

Dirección General de Ciudadanía Intercultural, Ministerio de Cultura keluzcg@hotmail.com

RESUMEN

Los cambios producidos en la sociedad en las últimas décadas y la creciente participación de las mujeres en el espacio público han impactado en el sistema de género y en los proyectos de vida de las mujeres. En este contexto, el articulo tiene como objetivo identificar y analizar las representaciones de maternidad de las mujeres jóvenes universitarias de estratos medios bajos de la ciudad de Lima y los significados atribuidos en la construcción de las identidades femeninas.

Desde un marco metodológico basado en la teoría del núcleo central y el desarrollo de entrevistas, se halla que las jóvenes entrevistadas planifican sus proyectos de vida, asentándolos en los ejes de maternidad y desarrollo profesional, pero con nuevas configuraciones. Estos tratan de ser integrados, pero se generan tensiones, aunque existe una mayor valoración de la maternidad frente a la participación en el espacio público. Principalmente, las jóvenes entrevistadas consideran que la maternidad constituye una fuente de afecto y compañia. Adicionalmente, la maternidad se basa en el sacrificio, se configura como el único espacio propio de las mujeres, constituye una idealización de la familia hegemónica y es una fuente de tensiones entre el espacio doméstico y espacio público.

Palabras clave: maternidad, jóvenes, trabajo, espacio doméstico, espacio público. 


\title{
Social representations of the motherhood of young women in Lima
}

\author{
ABSTRACT
}

The changes in society in recent decades and the growing participation of women in public space have impacted on the gender system and on women's life projects. In this context, the article aims to identify and analyze motherhood representations among among young female university students from lower to middle strata of Lima and the meanings attributed to the construction of female identities.

From a methodological framework based on the theory of the central nucleus and the development of interviews, we found that the young people interviewed plan their life projects based on factors such as motherhood and their professional development, but with new configurations. They try to integrate both of them, but tensions are generated, even though there is a greater appreciation of motherhood than of participation in the public space. Mainly, the young women interviewed consider that motherhood constitutes a source of affection and company. Additionally, motherhood is based on sacrifice, it is configured as the only space where women belong, it constitutes an idealization of the hegemonic family and it is a source of tensions between the domestic space and public space.

Keywords: motherhood, youth, job, domestic space, public space. 


\section{INTRODUCCIÓN}

En las últimas décadas, muchos cambios sociales, culturales, económicos y políticos han sucedido en el Perú y en todo el mundo, los que, sin duda, también han impactado en la vida de las mujeres. De esta manera, en la actualidad se han reducido múltiples brechas de género en el acceso a la educación y la participación en el espacio laboral y político, ha disminuido la tasa de fecundidad, cada vez más se retrasa la maternidad, se han generado cambios en las estructuras familiares, además del avance de discursos de autonomía de las mujeres e igualdad de género y el cuestionamiento de lo doméstico como único espacio de realización de las mujeres, entre otros importantes sucesos. Estos cambios han generado también modificaciones en el sistema de género, identidades femeninas y representaciones de la maternidad.

El cambio generacional y el mayor acceso de las mujeres a la educación universitaria han promovido una mirada más crítica al sistema tradicional de género y la construcción de proyectos de vida basados en la libertad y la autonomía. Además, ambos factores estarían influyendo en que la maternidad sea postergada con relación al desarrollo profesional, dimensiones que otorgan reconocimientos y gratificaciones a las mujeres. Adicionalmente, las mujeres que acceden a estudios superiores tienen un menor número de hijos en comparación con las que no tienen acceso a ellos. En ese contexto, las mujeres empiezan a reflexionar sobre la maternidad como obligación y no como una decisión más autónoma.

Específicamente, el mayor acceso de las mujeres a la educación universitaria estaría generando una mayor participación en el espacio público, lo que es valorado también en sus planes de vida. No obstante, se generarían tensiones en el proceso de articular e integrar su participación en el espacio doméstico y el espacio público. 
Además, con los cambios producidos en cada generación, las jóvenes tendrían cambios en sus imaginarios culturales acerca de la autonomía y empoderamiento de las mujeres en comparación con generaciones anteriores. Por otro lado, su procedencia de familias de estratos medios bajos generaría que sus padres y madres las impulsen a acceder a la educación universitaria y desarrollar una vida profesional para lograr buenos empleos y mejorar sus condiciones socioeconómicas y las de sus familias.

Desde el enfoque de género, el artículo analiza las representaciones de maternidad de las mujeres jóvenes universitarias de estratos medios bajos de la ciudad de Lima y los significados atribuidos en la construcción de las identidades femeninas.

El artículo se basa en los hallazgos de la tesis de maestría de la autora, titulada Representaciones sociales de la maternidad y los significados que le asignan las mujeres jóvenes universitarias de estratos medios bajos de Lima Metropolitana en la construcción de las feminidades e identidades femeninas. La tesis fue sustentada en el año 2016 para optar el grado de magíster en Estudios de Género por la Pontificia Universidad Católica del Perú.

\section{MARCO METODOLÓGICO}

El tema planteado se abordó desde la investigación cualitativa. Se realizaron entrevistas grupales e individuales en las que participaron diecisiete estudiantes de diversas carreras de las dos universidades públicas más grandes de Lima y del país: Universidad Nacional Mayor de San Marcos y Universidad Nacional Federico Villarreal.

Se realizaron dos entrevistas grupales (una por cada universidad de procedencia) para obtener principalmente las representaciones comunes del colectivo, a partir de los consensos y diferencias expresadas por las participantes. En estas entrevistas participaron siete estudiantes en total. Posteriormente se realizaron diez entrevistas individuales (cinco por cada universidad) para profundizar y ampliar los hallazgos a partir de las historias de vida de las estudiantes. Con este número, las entrevistas individuales alcanzaron el nivel de saturación de la información, ya que se empezaron a registrar patrones repetitivos acerca de las representaciones.

Casi todas las entrevistadas pertenecen a los estratos medios bajos, de acuerdo con la tipología de Ipsos Apoyo (2013). Para las entrevistas grupales e individuales se definieron las siguientes características: 
- Ser mujer.

- Ser joven: tener entre 15 y 29 años.

- Ser estudiante de universidades nacionales.

- Ser estudiante de los últimos años de carrera.

- Pertenecer al estrato medio bajo de Lima Metropolitana.

- No ser madre.

El marco metodológico se sostuvo principalmente en la teoría del núcleo central de Abric (2001), quien plantea que toda representación social está constituida por un núcleo central y elementos periféricos. El núcleo central está constituido por uno o varios elementos que determinan la significación y organización de la representación. Los elementos periféricos se refieren a los que se organizan alrededor del núcleo central, el cual define sus valores y funciones.

\section{IDENTIDADES Y SISTEMA DE GÉNERO}

Según Hall (2003), la identidad es una construcción en constante proceso de cambio y transformación opuesta a la noción de una identidad integral y unificada. La sociedad configura un repertorio de identidades que los individuos van reconociendo e internalizando a medida que son socializados, según plantea Berger (1982). En ese proceso de reapropiación, el sujeto construye sus representaciones de los espacios privados y públicos, especialmente las representaciones de la maternidad y el lugar que se le atribuye como configuración dentro de su identidad femenina y de las feminidades.

Asimismo, las identidades de género están enmarcadas en sistemas de género que se configuran también como estructuras dinámicas. Scott (1990) plantea que el género es un elemento de las relaciones sociales basadas en las diferencias de los sexos, constituido por los símbolos, normas, sistema institucional e identidad subjetiva. También expresa que el género va más allá de los cuerpos y se convierte en una forma primaria de relaciones significantes de poder, es decir, se establece una jerarquía entre lo que representa lo masculino y lo femenino.

Lamas (2002) expresa que en la identidad del sujeto se articulan subjetividad y cultura, lo psíquico y lo social, historias personales y vivencias según otras categorías, como clase, etnia, edad. Todos estos elementos operan de manera diferente, por ello, el sujeto está en permanente construcción. Asimismo, afirma 
que las normas de género constituyen un filtro cultural y la armadura que constriñe a los sujetos, ya que plantea comportamientos y actitudes en los diversos ámbitos a partir de una diferencia sexual.

Las normas de género se asientan sobre una base sexual binaria y jerárquica, que define características diferentes para hombres y mujeres, según las jóvenes entrevistadas. Las jóvenes entrevistadas consideran que hay diferencias biológicas. El embarazo y parto constituyen capacidades biológicas de las mujeres que marcan diferencias con respecto a los hombres y que son consideradas por las jóvenes entrevistadas como ventajas. De allí que se resalta a la maternidad como único espacio propio de las mujeres y se enfatiza la relación entre mujer y naturaleza. A su vez esto se asocia a que el espacio doméstico es importante - $y$ propio - para las mujeres por mandato natural.

No obstante, entre los significados atribuidos a las identidades femeninas por las jóvenes entrevistadas destacan la capacidad de agencia (fuerza, realización, lucha, autonomía y libertad) y un distanciamiento de la pasividad.

Las representaciones de las identidades femeninas de las entrevistadas están asentadas en dos dimensiones: por un lado, fuerza e independencia económica, y por otro, el afecto y cuidado enmarcado en la maternidad y familia. Es decir, se les reconoce el espacio doméstico como espacio propio, pero también reconocen que su realización se encuentra en el ámbito público.

Las jóvenes entrevistadas definen que una mujer realizada tiene dos dimensiones. Primero, es una mujer trabajadora, preferentemente con estudios superiores, su actividad laboral le genera satisfacción y logra resultados eficientes y óptimos; además, con ello obtiene independencia económica. La otra dimensión de la mujer realizada es tener una pareja y ser madre. Se enfatiza en que la mujer realizada es una mujer feliz, lo que implica estar satisfecha con lo que hace y tener una elevada autoestima.

Las jóvenes planifican y construyen un proyecto de vida que orienta sus prioridades y metas y no pretenden dejar sus objetivos al azar o al destino. Las jóvenes tienen como meta inmediata concluir la universidad, cursar estudios de posgrado - si es posible en el extranjero-, estabilizar su carrera laboral, tener espacios de disfrute como viajar, posteriormente consolidar una relación afectiva, especialmente dentro de un matrimonio, aunque la convivencia se presenta también como opción válida, y tras el cumplimiento de estas metas, ser madres. Este es el orden para que las dimensiones de desarrollo profesional y afectiva/ maternal sean asumidas con responsabilidad y aceptación social. 
En una mujer la realización se da en varios aspectos. Primero, profesional. Una persona realizada se ama mucho a sí misma, tiene mucha autoestima; también en el aspecto laboral, le va bien, está estable económicamente y ha formado una familia. Creo que la mayoría de mujeres con todas esas cosas se pueden sentir realizadas (Estela, 22 años, UNFV).

Se presentan cambios en el proyecto de vida de las mujeres entrevistadas en comparación con la generación anterior. Al matrimonio y a la maternidad como metas se les suma el desarrollo profesional. Aun cuando la mayoría de madres de las entrevistadas tienen estudios superiores, las jóvenes plantean retrasar su maternidad y tener un número menor de hijos que el que tuvieron sus madres. En esta generación también se visibiliza la búsqueda de relaciones de género más igualitarias; además, se evidencia que las mujeres construyen diversos proyectos de vida.

Estas diferencias intergeneracionales son abordadas por Fuller (2001), quien plantea que las identidades femeninas asentadas solo en la maternidad están sufriendo cambios, pero que estos no se dan de manera similar en todas las mujeres. La clase, edad, orientación sexual, etnia, además de otros factores como niveles de ingreso, educación, participación política, relaciones familiares y de pareja, plantean diversidad de nociones sobre la maternidad.

Los agentes de socialización (familia, escuela, medios de comunicación, iglesia y grupo de pares) tienen discursos que impulsan mayor autonomía, pero a la vez también son contradictorios y plantean ideas tradicionales sobre la feminidad, donde la presión por maternidad se presenta como un eje importante para la realización.

De acuerdo con lo hallado, los agentes de socialización incentivan el empoderamiento y autonomía en las jóvenes solo en el plano económico, aunque este constituye un importante avance para el proceso de un mayor y real empoderamiento.

Las jóvenes tuvieron la intención de estudiar en la universidad desde que eran niñas, pero en especial desde que eran adolescentes. Este deseo fue impulsado principalmente por las madres, pero también por los padres, parientes, docentes y amistades. Las entrevistadas consideran que el acceso a la educación superior es un camino al logro de la autonomía económica, que implica obtener un trabajo más estable para mejorar sus condiciones de vida y las de sus familias. Bajo esta idea también se encuentra que no generen relaciones de dependencia económica con sus parejas y promuevan relaciones de género más igualitarias. 
La familia constituye el primer espacio de socialización y construcción de las identidades de género. La familia inculca en las mujeres su relación con los espacios domésticos y públicos, es decir, cumplir un doble rol. Se evidencia que en las familias de las jóvenes hay una visión de familia tradicional, instituida mediante el matrimonio, que se espera que las hijas e hijos perpetúen. La figura materna es valorada por su lucha y sacrificio por su familia, así como su afán emprendedor por mejorar la economía familiar. No obstante, a la vez, es percibida como un sujeto sensible y que necesita protección de su pareja masculina.

En la escuela también se incentiva la autonomía económica de las mujeres y se les presenta el acceso a la educación superior como meta. De igual manera, las jóvenes tienen como tema central con sus pares el desarrollo profesional, puesto que se encuentran cerca de concluir la universidad. Eso implica que tienen una amplia expectativa por forjarse una trayectoria profesional sobresaliente.

La Iglesia promueve un modelo de mujer tradicional que es criticado por varias de las jóvenes entrevistadas, especialmente por las que no se autorreconocen como evangélicas. La Iglesia no alienta la libertad ni autonomía de las mujeres sino más bien el sacrificio y la culpa, además de la virginidad hasta el matrimonio. También se cuestionan las desigualdades de género existentes dentro de la Iglesia. Es visible la demanda de que la Iglesia se adecue a los tiempos actuales, cambios generados y derechos reconocidos de las mujeres.

Los medios de comunicación y la publicidad son criticadas por las jóvenes, pues consideran que presenta roles tradicionales de género. Expresan que a las mujeres se las representa solo como objetos sexuales, sujetos del ámbito doméstico y no se fomenta su participación en los espacios públicos.

Por otro lado, la sexualidad es un campo donde permanecen prejuicios en los diversos actores sociales, incluidas las mismas jóvenes, especialmente el ejercicio libre de la sexualidad de las mujeres en comparación con sus pares masculinos.

Los padres y madres resguardan celosamente la sexualidad de las hijas para evitar que tengan un embarazo no deseado y trunquen sus proyectos de vida. Pero este cuidado hacia la sexualidad de las hijas también connotaría el cuidado del vínculo entre pureza y feminidad, prestigio y valor.

La escuela se presenta como un espacio bastante conservador para las relaciones de género, puesto que plantea una división sexual del trabajo. También se configura como un espacio que busca controlar los cuerpos y sexualidades de las jóvenes.

En el grupo de pares, el cuidado de la apariencia física es también un tema central en las conversaciones bajo el argumento de la autoestima, bienestar y 
cuidado de sí misma. La apariencia física implica también construir una imagen de mujer dentro de los cánones esperados: delicadeza y pureza.

León (2013) expresa que en la actualidad las jóvenes presentan diversas actitudes con respecto a la sexualidad y roles de género: algunas jóvenes los aceptan y otras los rechazan. Esto genera tensiones entre, por un lado, el conservadurismo y la resistencia a desafiar públicamente estos modelos tradicionales de género, y por otro, la existencia de nuevos discursos de liberación femenina que cuestionan roles tradicionales y valoran el disfrute de la sexualidad. Esta diversidad de valores coexiste y se expresa según el espacio de interacción.

En general, se evidencia un discurso en las jóvenes que fluctúa entre lo conservador y lo moderno. Los agentes de socialización plantean argumentos que se contradicen entre sí y en varios casos son criticados por las jóvenes, en especial los discursos de la escuela, medios e Iglesia. Sobre ello, las dimensiones de la maternidad y el desarrollo profesional están presentes en los proyectos de vida de las jóvenes entrevistadas, pero con nuevas configuraciones.

\section{REPRESENTACIONES SOCIALES DE LA MATERNIDAD}

\section{Nociones sobre la maternidad}

La maternidad ha sido interiorizada en la construcción de las feminidades como un mandato natural, una práctica obligatoria y normalizada que tiene que estar en el plan de vida de las mujeres sin cuestionamientos ni reflexiones.

A partir de esta idea, se ha buscado definir la relación de identificación de las mujeres con la naturaleza y se ha considerado que ellas son seres con leyes biológicas que no les permiten espacio para la libertad de decisión, aun cuando la sociedad plantee que las mujeres deciden su maternidad de manera voluntaria.

En ese marco, Badinter (1981) afirma que el instinto materno es un mito que no responde a procesos naturales. Lo mismo sucede con las características que se le relacionan, como la responsabilidad, el amor, etc. Expresa que el amor maternal es un sentimiento y, como tal, es frágil, variable, imperfecto y puede no existir o desaparecer tras un tiempo.

Beauvoir (1999) cuestiona que las mujeres consideren la maternidad como su devenir natural y esté asociada a gratificaciones, satisfacciones y realizaciones femeninas, cuando en realidad constituye un mandato social que mutila la libertad de las mujeres, se presenta una fuerte dependencia y posesión con los hijos e 
hijas y les genera frustraciones porque no pueden vivenciar la maternidad como lo desean sino bajo el modelo único que establece el sistema social.

Tubert (1996) plantea que la maternidad tiene una base biológica, pero va más allá: «La maternidad no es puramente natural ni exclusivamente cultural; compromete tanto lo corporal como lo psíquico, consciente e inconsciente; participa de los registros real, imaginario y simbólico» (p. 13). Es decir, la maternidad se construye a partir de una articulación entre la psiquis, el cuerpo y la cultura. Asimismo, cuestiona que las representaciones de la maternidad tengan un poder reductor (se busca que todas las mujeres tengan como objetivo ser madres y esta experiencia es presentada como grata de manera magnificada sin evidenciar su complejidad) y uniformadora (se plantea que las experiencias de maternidad son iguales, con lo que se desconoce las identidades diversas de las mujeres).

Adicionalmente, Tubert (2007, p. 209) expresa: «Lo que debe recategorizarse es la noción de mujer como sujeto y no como mero sustrato corporal de la reproducción o ejecutora de un mandato social o encarnación de un ideal cultural». Es decir, la mujer, aun cuando sea madre, debe seguir manteniendo su condición de sujeto mujer y no ser reemplazado por el sujeto madre. En ese sentido, Donath (2016) establece que el cuerpo de las mujeres no debe ser juzgado por su capacidad o no para la reproducción, ya que deja de lado otras dimensiones del sujeto.

La investigación halló que la maternidad constituye un eje importante en las identidades femeninas y está incorporado en los proyectos de vida de las jóvenes universitarias de estratos medios de Lima Metropolitana.

En comparación con sus madres, quienes tuvieron a sus hijas alrededor de los veinte y veinticinco años, las jóvenes plantean su maternidad ideal como un estado que se alcanza alrededor de los treinta años, luego de fortalecer su desarrollo profesional y tener una base económica sólida, en el marco de un matrimonio o relación estable de pareja. Además, se idealiza tener alrededor de dos hijos para prodigarles suficiente tiempo y dedicación. El factor económico tiene mucha importancia cuando se aborda la maternidad, puesto que implica dar mejores condiciones de vida a la prole.

[...] Mi proyecto sería terminar mi carrera, sacar mi título profesional, seguir una maestría, una especialización, un doctorado, ubicarme en el cargo laboral en un buen puesto de trabajo que sea reconocido en una buena institución, en mi ámbito laboral que me vaya bien y casarme, tener una familia, un hogar en el cual me pueda comprender con mi esposo, que ante un problema podamos solucionarlo y no irnos a la violencia o pelear con nuestros hijos, tener un hogar tranquilo (Patricia, 21 años, UNFV). 
Entonces, la maternidad se encuentra en el proyecto de vida que se espera de las mujeres, es un proceso de «avanzar en el curso de la vida» (Donath 2016, p. 44). La maternidad está presente en el plan de vida de progreso, en la que constituye una puerta de ingreso a una vida mejor y un nuevo mundo (Donath, 2016).

Las jóvenes consideran que la maternidad genera profundos cambios positivos en las mujeres, les otorga muchas gratificaciones y potencia cualidades como la fortaleza, paciencia, dedicación y empatía. Donath (2016) expresa que la maternidad ha sido idealizada por la sociedad como el estado que generará madurez, consagración, satisfacción, y que será un estado donde la soledad se acabará.

En ese contexto, se destaca que el tema del aborto casi no fue mencionado por las jóvenes. Solo una joven expresó que proyectaron un video sobre el aborto, desde una mirada de culpa y rechazo, en su colegio religioso mixto.

Según la teoría de Abric (2001), la idea que constituye el núcleo central de las representaciones de la maternidad es que constituye una fuente de afecto y compañía. Como elementos periféricos se halla que la maternidad se basa en el sacrificio, se configura como el único espacio propio de las mujeres, constituye una idealización de la familia hegemónica y es una fuente de tensiones entre el espacio doméstico y el espacio público.

\section{Gráfico 1. Representaciones de maternidad de jóvenes universitarias de estratos medios bajos de Lima Metropolitana}

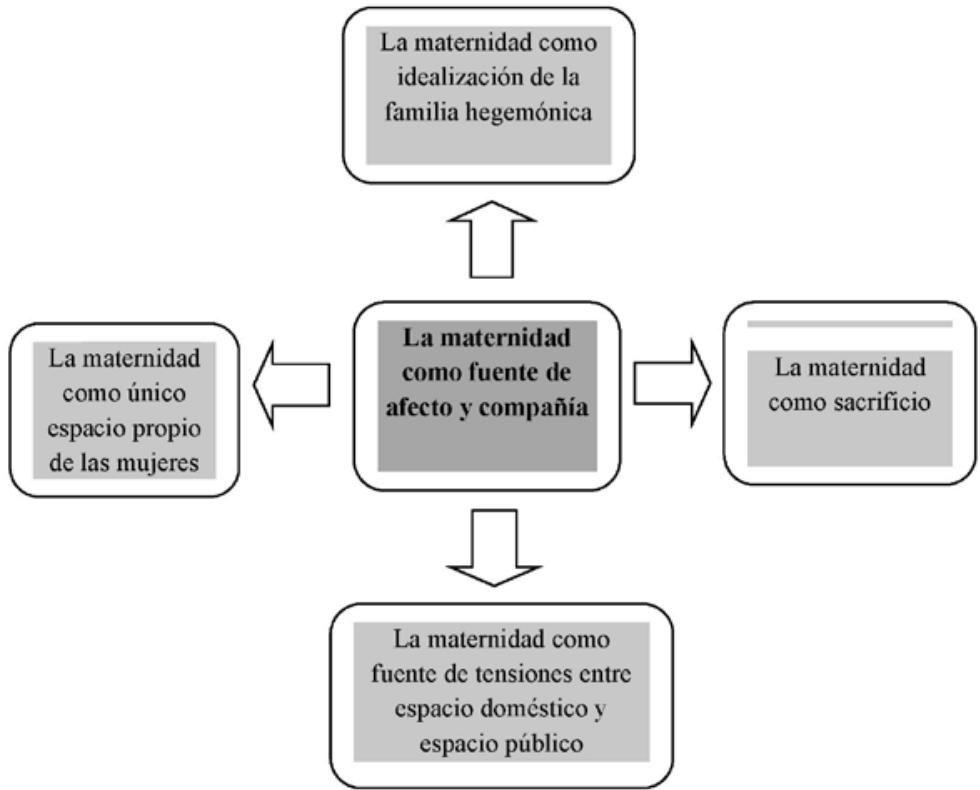




\section{La maternidad como fuente de afecto y compañia}

La maternidad es un eje central en la constitución de las identidades femeninas. Como núcleo central de las representaciones de la maternidad —es decir, la base común que define la significación y organización de la representación, según la teoría de Abric (2001) - la maternidad constituye una fuente de afecto y compañía.

La maternidad significa tener una «persona compañera» para toda la vida con el fin de no enfrentar la soledad. La madre será un sujeto necesario e importante para el otro, con quien construirá una relación de dependencia. Estas afectividades enfocadas en la maternidad no son satisfechas por la pareja ni por la familia.

¿Qué les da [la maternidad a las mujeres]? No sé, afecto distinto, o sea les da creo otro tipo de valoración en el hecho de que son aquellas las que pueden procrear y pueden tener al hijo, o sea son las indicadas o supongo también que las complementa, el hecho de tener un hijo para que las complemente también o las acompañe (Laura, 23 años, UNFV).

Asimismo, la mujer no es considerada como unidad, por lo que se piensa que la maternidad la convertirá en unidad, es decir, un hijo complementará al sujeto mujer para que sea un sujeto completo.

El hijo o la hija también se convierten en el sujeto que reproducirá muchos aspectos positivos de las madres; para ello, las madres deben transmitirle sus valores y saberes. De esta manera, se construye una relación de pertenencia y posesión de la madre hacia el hijo.

Para las jóvenes entrevistadas, la maternidad está asociada a la responsabilidad, es decir, implica hacerse cargo de una persona, cuidarla, atenderla, guiarla bajo los valores que considera importantes la madre y el padre. De manera específica, esta idea de cuidado del otro de acuerdo con las ideas y valores de la mujer es una de sus formas de «trascendencia»y «perduración».

Según Marcús (2006), la dependencia generada entre la madre y el hijo también implica que la maternidad sea una fuente de poder y reconocimiento; esto expresa que también la madre puede ejercer control sobre los hijos e hijas. El hijo necesitará constantemente de la madre, por lo que se construye una relación de necesidad que genera un poder para la madre y su legitimación como autoridad. Además, los hijos e hijas son sujetos que reproducen y afirman a la madre como dadora de identidad. 


\section{LA MATERNIDAD COMO SACRIFICIO}

El primer elemento periférico establece la noción de maternidad como sacrificio. Las representaciones de la maternidad se asientan, por un lado, en un modelo mariano, donde el sacrificio y la entrega a los hijos e hijas es parte central de las identidades femeninas, tal como precisa Fuller (1998). Incluso Beauvoir (1999) plantea que la maternidad se configura como un sacrificio en favor de la reproducción de la especie. Esta idea del sacrificio está presente en todos los discursos de las entrevistadas y también constituye un eje en la educación de las mujeres en el marco de la construcción de sus identidades.

En ese sentido, Palomar (2004) afirma que el modelo único de maternidad, que el sistema de género trata de imponer, invisibiliza al sujeto mujer y construye al sujeto madre. Con esto, la maternidad despoja al sujeto mujer de necesidades y deseos para priorizar la necesidad del hijo. La maternidad como sacrificio constituye una pérdida para las mujeres, un sacrificio de sí mismas y cierra la puerta a otros intereses o empresas personales, según Pariona (2016).

Surge como preocupación el tiempo que las madres tienen que dedicar a la crianza del hijo o hija bajo los parámetros que ellas consideran ideales, lo que se relaciona con estar presente o ausente en la crianza. En ese sentido, el sacrificio de la maternidad implica que la mujer tenga que dejar o detener su carrera laboral y profesional para ejercer su rol de cuidadora de sus hijos e hijas. Ese ha sido el caso de la mayoría de las madres de las jóvenes.

[Con la maternidad] ya no solo la mujer piensa en ella misma, ya también en sus hijos; económicamente también ya a veces, a veces, ni piensa en ella ya, sino solamente en los hijos, en parte del tiempo y todo eso, no es igual, es muy distinto (Alicia, 27 años, UNMSM).

El sacrificio también implica que la mujer se desexualiza. La maternidad convierte al sujeto mujer en un sujeto asexual, sin deseos, ni erotismo y refuerza la idea de la sexualidad con fines reproductivos (Garay, 2008). La madre no se reconoce como sujeto con derecho a tener sus emociones y eso genera mayores culpas, según Donath (2016).

El sacrificio tiene también una dimensión biológica con la menstruación, el malestar del embarazo y el dolor de parto. El dolor de estos estados también conforma el sacrificio que deben enfrentar las mujeres. 


\section{LA MATERNIDAD COMO ÚNICO ESPACIO PROPIO DE LAS MUJERES}

Precisamente, el segundo elemento periférico es la idea de la maternidad como único espacio propio de las mujeres. En esa línea, el embarazo y el parto constituyen capacidades biológicas de las mujeres que marcan diferencias con respecto a los hombres, lo que es considerado por las jóvenes entrevistadas como una ventaja frente a los varones.

Yo creo que el hecho de decidir tener un niño es porque se siente bonito dar la vida, como mujer, que no lo puede hacer el varón, es decir sin complemento, el hecho de darle la vida a una persona y darle todo lo mejor que uno tiene y puede, creo que es algo satisfactorio, el hecho de compartir tu tiempo, es también tener más responsabilidades pero es también el hecho de disfrutar, compartir y darle lo mejor a esa persona (Gabriela, 24 años, UNFV).

Según las entrevistadas, la maternidad se inicia con el embarazo. Esta dimensión biológica de la maternidad resulta satisfactoria, pero a la vez genera temor, debido a los procesos, cambios y riesgos que sufren los cuerpos de las mujeres. La mortalidad materna y malformaciones genéticas en los hijos e hijas también generan temor, al igual que la infertilidad femenina.

En esa línea, el embarazo y el parto tienen un fuerte componente simbólico en la maternidad y en el vínculo de afecto entre la madre y el hijo. La maternidad biológica se presenta como un estado de autoridad y poder, puesto que la mujer albergó al hijo en su propio organismo. Esto coincide con lo que señala Garay (2008) acerca de la valoración de los lazos biológicos como base de las relaciones de parentesco, idea que también es reforzada por las nuevas tecnologías reproductivas. Este discurso genera que se desvalorice la maternidad por adopción, pues se considera que no se construye el mismo lazo entre madre e hijo, aunque fue un tema que no surgió explícitamente en las entrevistas.

Las jóvenes creen que todas las mujeres tienen instinto materno, lo que acentúa su idea de la maternidad como un devenir natural. En esa línea, las jóvenes creen que todas las mujeres, sean madres o no, «de todas maneras» cuidarán a otras personas. No obstante, Badinter (1981) fundamenta que el instinto materno es una construcción cultural, al igual que el amor materno. Expresa que este ha sido sobredimensionado bajo el argumento de que tiene una base biológica.

La idea de las jóvenes acerca de la relación entre mujer y naturaleza basada en la condición biológica de la maternidad estaría asociada a que el espacio doméstico es importante - y propio - para las mujeres, por mandato natural. No obstante, las jóvenes consideran que los hombres también deben participar en este espacio, sobre todo cuando son padres. 


\section{LA MATERNIDAD COMO IDEALIZACIÓN DE LAFAMILIAHEGEMÓNICA}

Jelin (1998) indica que la familia es la organización social que alberga relaciones de producción, reproducción y distribución, así como relaciones de poder y componentes ideológicos y afectivos como base de su organización, las que permiten su subsistencia y reproducción.

En esa línea, el tercer elemento periférico de las representaciones de la maternidad es que responde a una idealización de la familia hegemónica. El modelo de maternidad se enmarca dentro de una familia tradicional y heteronormativa por los componentes que reúne: fertilidad, heterosexualidad y pareja (Burgaleta, 2011).

Sí creo que sería lo más ideal, lo más bonito, lo más genial, tener una pareja con quien poder afrontar todas las cosas que puedan suceder dentro del hogar, aquellas adversidades, económicas, problemas con los hijos o cosas así. Sería bonito (Amaya, 22 años, UNMSM).

Precisamente, las jóvenes explicitan su deseo de ser madres dentro de una relación estable de pareja y el matrimonio como institución en un contexto ideal. No obstante, algunas entrevistadas consideran legítima la convivencia con lo que el matrimonio no es planteado como esencial para ser madre. La maternidad define el vínculo heterosexual bajo la idea de la complementariedad con bases biológicas, donde la pareja tiene roles de género definidos y cada uno aportará un modelo de identidad a los hijos e hijas.

La pareja no representa una importante fuente de afecto para las mujeres entrevistadas, puesto que esta función es atribuida a los hijos e hijas. En ese sentido, las jóvenes no plantean como prioritaria la dimensión afectiva como pareja, sino que se presenta un pragmatismo en las relaciones afectivas. La pareja se configura como un sujeto necesario para hacer posible la maternidad y para proveer cuando la mujer deje de trabajar y se dedique a la crianza de los hijos e hijas.

Las jóvenes demandan que los hombres participen en el espacio doméstico, en especial cuando son padres. Esto significaría un avance en la igualdad de participación en el ámbito doméstico que se plantea la generación joven, pero esta participación aún es reducida; además, no se avizora una participación tan activa como la de las madres.

Pocas entrevistadas relatan la posibilidad de la maternidad en soltería, puesto que los estigmas y estereotipos sobre las madres solteras subsisten en el imaginario social. Además, afirman que la dimensión económica genera que esta situación no sea percibida como posible. 


\section{LA MATERNIDAD COMO FUENTE DE TENSIONES ENTRE EL ESPACIO DOMÉSTICO Y ESPACIO PÚBLICO}

El cuarto elemento periférico de las representaciones de las jóvenes entrevistadas es que la maternidad constituye una fuente de tensiones entre el espacio doméstico y el espacio público, además que explicita el doble rol que desarrollan las mujeres y que buscan equilibrar como ejes centrales de sus identidades.

[El trabajo otorga a las mujeres] autonomía, dependencia, también desarrollo, implica desarrollarse, autonomía también, porque una tiene un trabajo estable, tiene ingresos, creo que no va a depender de nadie para realizar cualquier cosa que desee realizar (Graciela, 20 años, UNMSM).

Las jóvenes expresan que el trabajo otorga a las mujeres elementos importantes para su valoración como sujetos: la independencia económica y el desarrollo de sus habilidades y capacidades, además que las convierte en sujetos que aportan a la sociedad. Pariona (2016) plantea que el trabajo es un aspecto fundamental en la construcción de las identidades y uno de los principales vectores para entender el proyecto de vida de mujeres profesionales. En esa línea, Jelin (1998) plantea que la participación de las mujeres en el espacio laboral se incrementa en cada generación, especialmente en las mujeres de clase media y que acceden a la educación superior. Es decir, no se detendrá el ingreso de las mujeres al espacio laboral, aun más si acceden a la educación superior.

El logro de la independencia económica es bastante valorado por las jóvenes, puesto que generaría una mayor igualdad en sus relaciones con los hombres, especialmente en el marco de las decisiones que tomen con sus parejas. Además, las madres de las jóvenes las impulsan a lograr esta independencia que se relaciona con el interés de que accedan a una educación superior universitaria.

Las jóvenes están retrasando la maternidad para lograr las condiciones para ser madres y fortalecer su participación en el espacio público, pero también es el logro de realizaciones de otras metas, como plantea Pariona (2016). No obstante, las jóvenes no están negando la maternidad, puesto que se presenta como una dimensión diferente pero complementaria del desarrollo profesional. En esa línea se encuentran también los hallazgos de Fuller (1998) y Castro (2005).

Las entrevistadas consideran importante equilibrar ambos proyectos, aunque piensan que se presentan periodos en los que se tiene que priorizar uno de los dos ámbitos. Además, opinan que las mujeres no pobres lograrán de mejor manera el logro de este equilibrio. 
Precisamente, tratar de lograr el equilibrio entre la maternidad y su carrera laboral y profesional genera que las mujeres cumplan una doble jornada, lo que conlleva a un mayor esfuerzo y tener poco tiempo para sí mismas. Esta también sería una expresión del sacrificio de la maternidad y también de la figura idealizada de la súper mujer, figura poco real de lograr que a las mujeres les genera una tensión constante, un exagerado y poco beneficioso esfuerzo físico y mental y un sentimiento de culpa.

Las jóvenes consideran que la maternidad implicaría hacer una pausa en sus carreras profesionales. En sus proyectos de vida, las jóvenes indican que quisieran dedicarse a ser madres a tiempo completo por un tiempo aproximado de un año o tres años, período en que consideran que el hijo necesita cuidado total de la madre y es la edad en que ya dejaría el espacio familiar para ingresar al sistema escolar. Esta situación ha sido realizada de manera similar por casi todas las madres de las jóvenes entrevistadas; además, también responde a los hallazgos de Castro (2005) y Pariona (2016) en mujeres adultas de clase media. No obstante, esto podría generar una dependencia económica de sus parejas, aun cuando esta situación ha sido bastante criticada e indeseada por las jóvenes.

Yo también creo que [una mujer deja su carrera laboral para dedicarse a cuidar a sus hijos] porque llegó un momento en que se sintió suficientemente realizada profesionalmente y buscó llenar, completar otro aspecto de su vida, o simplemente porque cuando ya fue madre, en ello pudo encontrar lo que no pudo obtener profesionalmente. No es lo mismo criar un hijo que tener una carrera o desarrollarse, todo eso, pero tal vez encontró despertar otros sentimientos en ella, por lo cual busca completamente dedicarse a ella (Amaya, 22 años, UNMSM).

Luego de esta pausa en la carrera laboral, las jóvenes indican que la retomarían, quizá en una menor jornada laboral, pero no la abandonarían porque reportan que es un espacio que también les genera satisfacciones, reconocimientos, y les aporta beneficios económicos. No obstante, algunas jóvenes respaldan la decisión de las mujeres que dejan su carrera profesional para dedicarse plenamente a su maternidad por tiempo indefinido. Consideran que esa dedicación hacia el niño generará que sea un «buen ciudadano», y ese será el aporte de la madre a la sociedad. En esa línea, se visibiliza la idealización de algunas jóvenes acerca del tiempo en que la gran mayoría de mujeres solo se dedicaban a lo doméstico.

Fuller (1998) y Castro (2005) hallan que las mujeres no pretenden abandonar el mundo doméstico, que les genera satisfacciones y reconocimiento, pero tampoco desean que sea su único espacio, por lo que buscan desarrollarse también en el 
ámbito público. Esto responde a que el espacio público puede constituir un espacio de reconocimiento, desarrollo y autonomía, mientras que el espacio doméstico representa un espacio de cuidado de sí mismas y del otro (Castro, 2005). En esa línea, las jóvenes también consideran que ambos espacios se retroalimentan y complementan.

En este marco, Beauvoir (1999) explica que el sistema de género define que la paternidad no genere cambios en la vida y libertad de los hombres, mientras que la maternidad genera que las mujeres renuncien a sus proyectos y se dediquen al espacio doméstico. Esto convierte a la maternidad en una limitación de la feminidad y un impulsador de la existencia de brechas de género.

\section{NO MATERNIDAD}

Las jóvenes están retrasando la maternidad, pero no la están negando. Sin embargo, también consideran que hay mujeres que deciden no ser madres y expresan actitudes de mayor comprensión y respeto acerca de estos proyectos de vida construidos de manera autónoma.

No obstante, se considera que mayoritariamente la no maternidad no es un estado que se decide, sino que es un devenir producto de muchas circunstancias, tales como problemas reproductivos, no tener una pareja o haber dedicado mucho tiempo a su trabajo.

Además, la no maternidad está asociada a muchos significados negativos. Entre los más comunes destacan la pena y lástima: las mujeres que no son madres - y más aún las que deciden no serlo - son consideradas como mujeres egoístas, con libertad, sin responsabilidades, dedicadas a su cuidado propio. No obstante, varias de estas representaciones son aceptadas por las entrevistadas mientras son jóvenes: valoran la autonomía, el cuidado de sí mismas. Cuando las mujeres son madres tienen que ser dependientes, dedicadas al otro, sacrificadas y sin mayores libertades. Esto evidencia el doble discurso que las jóvenes tejen acerca de los valores femeninos.

Sin embargo, algunas entrevistadas tienen apreciaciones más comprensivas y de respeto hacia las mujeres que deciden no ser madres, reconocen su libertad para decidir su proyecto de vida y evitan estigmatizarlas. Esto se enlazaría con la comprensión de la complejidad de la maternidad.

[La maternidad y la no maternidad] creo que son felicidades distintas, no podría compararlas (Juliana, 22 años, UNMSM). 
Yo creo que [la maternidad] depende de cada mujer, porque hay mujeres que han tomado la decisión de no ser mamás y son felices. Conozco varias tías que no sienten capacidades de tener responsabilidades tan grandes como educar, criar, mantener un hijo, entonces no los tienen y bueno, es su decisión. Pero también tengo compañeras [de la universidad] que cuando conversamos dicen que su sueño, el sueño de toda mujer es ser mamá, como que eso, eso sí creo que es un estereotipo social, relacionar la maternidad con lo femenino, con lo mujer, por ejemplo lo que escucho, «si no encuentro a un buen hombre, aunque sea voy hacerme inseminación y yo puedo criar a mis hijos sola, porque yo quiero ser mamá y no necesitaría un hombre para criarlos». Yo creo que ahí hay diversos tipos de ideas, hay mujeres que se sienten bien estando solas, sin hijos; hay mujeres que sí realmente quieren formar una familia feliz, estar con papá, mamá y sus hijos, y hay mujeres que bueno, quieren tener un hijo, pero no necesitan de un hombre. Entonces creo que eso depende de la ideología de la mujer, de su forma de pensar... Creo que una mujer con hijos es una mujer limitada, más consumida por alguien, [mientras que] una mujer sola es autónoma, creo yo (Samantha, 22 años, UNMSM).

La no maternidad decidida desafía este modelo hegemónico de realización de las mujeres planteado por el sistema de género tradicional, y considera que la mujer no solo es un sujeto biológico reproductivo, sino que puede ejercer su libertad para decidir sobre sí misma y, con ello, replantear el binomio mujer = madre y las dimensiones de la identidad femenina.

La sociedad es crítica con las mujeres que deciden analizar la maternidad como algo que puede no merecer la pena. La no maternidad genera estereotipos, sanciones y castigos, eso indica que es una vida «fuera de la norma impuesta a las mujeres» y que la maternidad no es elegida con libertad, plantea Donath (2006).

Según Burgaleta (2011), la no maternidad está relacionada con el sufrimiento ante la desobediencia del mandato tradicional de la maternidad, y una de las maneras para expiar la culpa es el cuidado a otras personas.

La no maternidad también se asocia al extremo del espectro de «mala madre», puesto que se presenta como una amenaza a la naturaleza femenina y como mujeres que no cumplen con ideales ni expectativas para ser madres (Palomar, 2004).

La no maternidad constituiría un cuestionamiento profundo al sistema binario y una afrenta a la familia hegemónica (Mojzuk, s/f). Sobre esto, Tubert (2007) indica que «lo que debe recategorizarse es la noción de mujer como sujeto y no como mero sustrato corporal de la reproducción o ejecutora de un mandato social o encarnación de un ideal cultural» (p. 209). 


\section{REFLEXIONES FINALES}

La investigación evidencia que la maternidad continúa siendo un eje central de las identidades femeninas y se configura como un espacio de gratificaciones y reconocimiento para las jóvenes universitarias de estratos medios bajos de Lima.

El desarrollo profesional también constituye una dimensión importante que tratan de articular con la maternidad y su participación en el espacio doméstico. Las jóvenes tratan de integrar estos dos ejes en sus proyectos de vida, aun cuando se generen tensiones, pero también tratan de resolverlos según sus prioridades de acuerdo con el contexto en el que se encuentran.

Las representaciones sociales muestran que existen cambios en el reconocimiento de las decisiones de las mujeres sobre sus historias de vida de manera más libre y autónoma, pero a la vez se evidencian tensiones con la permanencia de nociones estereotipadas que responden a un sistema de género tradicional.

Para superar estas tensiones, se evidencia la necesidad de construir relaciones más igualitarias entre hombres y mujeres con el fin de que tengan las mismas oportunidades de participación en el espacio doméstico y público. En ese sentido, la maternidad tiene que ser asumida como una función social que no compete solo a las madres.

Por último, se destaca la necesidad de visibilizar las diversas y reales experiencias de maternidad de las mujeres, que contrastan con la idealización que promueve el sistema social. Otro punto clave también constituye tener una mirada de respeto a la diversidad de decisiones de las mujeres sobre sus proyectos de vida, en especial cuando se decide por la no maternidad.

\section{REFERENCIAS}

Abric, Jean-Claude (2001). Prácticas sociales y representaciones. México, D. F.: Coyoacán.

Badinter, Elisabeth (1981). ¿Existe el amor maternal? Historia del amor maternal. Siglos XVII al XX. Barcelona: Paidós.

Beauvoir, Simone de (1999). El segundo sexo. Buenos Aires: Editorial Sudamericana.

Berger, Peter L. (1982). La identidad como problema en la sociología del conocimiento. En Gunter W. Remmling (ed.), Hacia la sociología del conocimiento. Origen $y$ desarrollo de un estilo del pensamiento sociológico (pp. 355-368). México, D. F.: Fondo de Cultura Económica. 
Burgaleta, Elena (2011). Género, identidad y consumo: las «nuevas maternidades» en España. Tesis de doctorado. Madrid: Universidad Complutense.

Castro Bernardini, María del Rosario (2005). Mujeres profesionales jóvenes: redefinición de roles tradicionales femeninos. Tesis de Licenciatura en Sociología. Lima: Pontificia Universidad Católica del Perú, Facultad de Ciencias Sociales.

Donath, Orna (2016). \#madres arrepentidas. Una mirada radical a la maternidad y sus falacias sociales. Barcelona: Reservoir Books.

Fuller, Norma (1998). Dilemas de la femineidad. Mujeres de clase media en el Perú. Tercera edición. Lima: PUCP.

Fuller, Norma (2001). Maternidad e identidad: relato de sus desencuentros. En Solum Donas Burak (comp.), Adolescencia y juventud en América Latina (pp. 235-242). Cartago: Libro Universitario Regional.

Garay, Ricardo (2008). El destino de ser madres: la ideología de la maternidad como soporte discursivo de las nuevas tecnologías reproductivas. En Mónica Tarducci (coord.), Maternidades en el siglo XXI (pp. 29-59). Buenos Aires: Espacio Editorial.

Hall, Stuart (2003). Introducción: ¿quién necesita «identidad»? En Paul Du Gay y Stuart Hall (comps.), Cuestiones de identidad cultural. Buenos Aires: Amorrortu.

Ipsos Apoyo (2013). Perfiles socioeconómicos de Lima Metropolitana 2013. Lima: Ipsos Apoyo.

Jelin, Elizabeth (1998). Pan y afectos. La transformación de las familias. Buenos Aires: Fondo de Cultura Económica.

Lamas, Marta (2002). Cuerpo: diferencia sexual y género. México, D. F.: Taurus.

León, Doris (2013). Feminidades en conflicto y conflictos entre mujeres. Género, transgresión y violencia entre mujeres adolescentes de dos colegios públicos de Lima. Lima: Senaju.

Marcús, Juliana (2006). Ser madre en los sectores populares: una aproximación al sentido que las mujeres le otorgan a la maternidad. Revista Argentina de Sociología, 4(7), 110-119. Buenos Aires. Consulta: 21 de marzo de 2016. http://www. redalyc.org/articulo.oa? $\mathrm{id}=26940705$

Mojzuk,Marta(s.f.).Entreelmaternalismoylaconstrucciónsociopolíticadelamaternidad. https:/patagonialibertaria.files.wordpress.com/2014/12/68101129-entre-elmaternalismo-y-la-construccion-socio-politica-de-la-maternidad-marta-mojzuk. pdf. Fecha de consulta: 16 de abril de 2014.

Palomar Verea, Cristina (2004). «Malas madres»: la construcción social de la maternidad. Debate Feminista, 15(30), 12-34. 
Pariona Icochea, Tania Edith (2016). Postergación de la maternidad en mujeres profesionales residentes en Lima. Tesis de Maestría en Antropología. Lima: Pontificia Universidad Católica del Perú, Escuela de Posgrado.

Scott, Joan (1990). El género, una categoría útil para el análisis histórico. En James S. Amelang y Mary Nash (eds.), Historia y género. Las mujeres en la Europa moderna y contemporánea. Valencia: Edicions Alfons El Magnanim, Institució Valencina d Estudis i Investigació.

Tubert, Silvia (1996). Introducción. En Silvia Tubert (ed.). Figuras de la madre (pp. 7-37). Madrid: Universidad de Valencia, Instituto de la Mujer.

Tubert, Silvia (2007). Maternidad. En Susana Beatriz Gamba (coord.), Diccionario de estudios de género y feminismos (pp. 208-210). Buenos Aires: Biblos. 